\title{
Zöhrer, Michaela (2020): Repräsentation ferner Wirklichkeiten. Umstrittene Wissensproduktion in wissenschaftlicher und humanitärer Praxis
}

Baden-Baden: Nomos. 388 Seiten. $79 €$

\author{
Katja Freistein
}

Angenommen: 23. September 2021 / Online publiziert: 14. Oktober 2021

(C) Der/die Autor(en) 2021

Nicht zuletzt durch die größere Aufmerksamkeit für postkoloniale und feministische Ansätze, die langsam in der Mitte der Sozialwissenschaften angekommen sind, bekommt die Positionalität von Wissenschaftler*innen und damit verbundene Fragen von Repräsentation mehr Gewicht. In ihrer beim Nomos-Verlag erschienenen Doktorarbeit „Repräsentation ferner Wirklichkeiten. Umstrittene Wissensproduktion in wissenschaftlicher und humanitärer Praxis“ geht Michaela Zöhrer daher Fragen nach, wie (wissenschaftliche) Repräsentationspraxis und Erkenntnisgewinnung mit fernen Subjekten/Objekten umgehen sollten. Angesichts der (scheinbaren) Nähe von Krisen, wie der in Echtzeit ablaufenden Eroberung Afghanistans durch die Taliban und Evakuierung gefährdeter Afghan*innen aus Kabul, sind wir uns der Schwierigkeit, diese angemessen erfahrbar zu machen, schmerzlich bewusst. Diese Schwierigkeit präsentiert sich als zentraler Gegenstand der Studie.

Die Arbeit von Zöhrer fügt sich damit nahtlos in ein transdisziplinäres Forschungsfeld ein, in dem insbesondere in den Internationalen Beziehungen und der Humangeografie Arbeiten entstanden sind, die sich kritisch mit der bildlichen Annäherung und damit verbundenen Reproduktion von humanitären Katastrophen oder Armut befassen. Auch an aktuelle Vorstöße zu Reflexivität in der wissenschaftlichen Praxis und den Umgang mit dem Anderen schließt sie produktiv an. In ähnlicher Weise wie viele Wissenschaftler*innen, die sich als kritisch verorten, geht es auch Zöhrer darum, mit welchen Maßstäben die Annäherung an das „ferne“ Subjekt zu bewerten oder rechtfertigen sein soll und was diese Annäherung bewirkt, vor allem mit Blick auf unser auch wissenschaftliches Wissen über die Welt: „Wie und aus welcher Position heraus wird etwas oder jemand als fern und/oder als fremd, an-

Katja Freistein $(\square)$

Centre for Global Cooperation Research/Käte Hamburger Kolleg, Universität Duisburg-Essen,

Duisburg, Deutschland

E-Mail: freistein@gcr21.uni-due.de 
ders, eventuell sogar als abnormal oder defizitär ausgewiesen, hervorgebracht und bedeutsam?“ (S. 27). Denn Repräsentation kann auch, wie Zöhrer diskutiert, selbst zur Intervention werden, wenn die Differenz zum Anderen bewertet wird und damit implizite Hierarchien entstehen, die wiederum in der Praxis zum Ausdruck kommen, z. B. als „Repräsentationspolitik“ (S. 221). Der kritische Blick etwa auf die Repräsentationspraxis humanitärer Nichtregierungsorganisationen, den Zöhrer hier nachvollzieht, ist in den Internationalen Beziehungen ja bereits etabliert, wenn auch die Auswege für moralische und pragmatische Dilemmata dieser Akteure fehlen.

Zöhrer verbindet fruchtbar unterschiedliche Wissensbestände, etwa aus der politischen Theorie, der Kulturwissenschaft und der Wissenssoziologie sowie diskursund praxistheoretische Perspektiven, um das Problemfeld Repräsentation von unterschiedlichen Seiten zu beleuchten. In dieser Zusammenschau werden die aktuellen Diskussionen anschaulich miteinander in Beziehung gesetzt und gewinnbringend in neue Fragen übersetzt. Diesen Fragen geht die Studie, die im Wesentlichen wissenssoziologisch bestehende Wissensbestände aufarbeitet, in zwei miteinander verwandten Betrachtungen am Untersuchungsgegenstand nach. Aus der historisierenden Rekonstruktion, wie humanitäre Notlagen repräsentiert werden, ergibt sich der Befund einer Kontinuität aus der kolonialen Tradition heraus bis heute. Zöhrer zeigt dies an der spezifischeren Auseinandersetzung mit dem Subjekt „Hungerkind“, das in Wissenschaft und Praxis schon länger als Gegenstand problematisiert wird, aber allen Bemühungen zum Trotz nicht von egalitären, weniger exkludierenden Formen der Repräsentation abgelöst wird.

Obwohl oder vielleicht gerade weil die Arbeit sehr umfassend viele wichtige Themen beleuchtet, wirft sie - wie kaum anders möglich - weitere Fragen auf. Zum einen sind das Fragen nach den politischen Lehren, zum anderen wissenschaftsinterne Verknüpfungen. Gerade mit Blick auf unterschiedliche aktuelle humanitäre Krisen, von denen einige sichtbarer sind als andere, bleiben auch drängende Fragen offen, die den Blick eher auf die Wirkung von Repräsentationen als auf Positionalität lenken. Wie hängt beispielsweise die weitgehende Unsichtbarkeit der Hungerkatastrophe im Jemen mit falscher, fehlender, angemessener Repräsentation zusammen? Können solche Krisen durch Selbstrepräsentation der betroffenen Subjekte besser als durch die mangelhafte Repräsentation durch Nichtregierungsorganisationen Aufmerksamkeit erzeugen? Wenn man sich vor allem für eine angemessene Darstellung von außen ausspricht, kann dann die humanitäre Krise überhaupt legitim zum Subjekt von Repräsentation werden - und wenn nicht, was bedeutet dies dann politisch?

Aus der Binnenperspektive der Wissenschaft gesehen, stellen sich noch einmal andere Fragen. Einige Verknüpfungen mit neueren Forschungsschwerpunkten der Internationalen Beziehungen werden angerissen, aber nicht vertieft - hier wäre über die Studie hinaus Potenzial für eine Weiterentwicklung der Argumente. Dazu gehört etwa das unter anderem in feministischen und normenzentrierten Ansätzen gestärkte Feld der Emotions- bzw. Affektforschung, die im Buch kurze Erwähnung findet. Die affektive Dimension von Bildern und Mobilisierung von Gemeinschaften oder (politischen) Reaktionen kann ein wichtiger Mechanismus in der Repräsentation humanitärer Notlagen sein, der sich eben nicht auf individuelle Befindlichkeiten reduzieren lässt, sondern kollektive Handlungen prägt. Dies ist oft auch in der Forschung zu Visualisierung erkannt und systematischer berücksichtigt worden, die 
mit eingehenden Bildanalysen oder quantitativen Studien zu Bildkorpora interpretativ ähnliche Widersprüche, Machtverhältnisse oder diskursive Schließungsversuche aufdeckt, wie sie Zöhrer beschreibt. Schließlich kann man ähnliche Kritik wie in Zöhrers Buch auch an der Repräsentation nicht so ferner Wirklichkeiten üben, die ebenfalls erwähnt werden. Denn auch diese kann Differenz, Hierarchien und normativ problematische Deutungen erzeugen und nähren, wie beispielsweise in der Repräsentation ,,prekärer“ Verhältnisse oder von Migrant*innen deutlich wird.

In der sehr verdichteten und reflektierten Annäherung an die Repräsentation ferner Subjekte und Zustände als Herausforderung für Wissenschaft und Praxis sind aktuelle Wissensbestände aus unterschiedlichen Disziplinen und Zugängen für Leser*innen auch mit wenig vorherigen Kenntnissen systematisch aufgearbeitet und daher gewiss von Interesse, gerade im Anschluss an die Forschung zu Entwicklung, Frieden und Konflikt, Nichtregierungsorganisationen oder sogar, sehr weit gefasst, dem Globalen Süden. Für wissenssoziologisch, diskurs- und praxistheoretisch orientierte Forscher*innen und solche, die auch mit feministischen und postkolonialen Perspektiven vertraut sind, denen die genannten Probleme aus der eigenen Arbeit daher geläufig sein dürften und die mit der einschlägigen Literatur bereits vertraut sind, ist vor allem die synthetisierende Diskussion unterschiedlicher Ansätze bereichernd, die viele Stränge verbindet und abwägt. Den in die Wissensproduktion selbst involvierten Leser*innen können die Einsichten über unsere Verwicklung in die problematische Repräsentation dieser ,fernen Wirklichkeiten“ einen Denkanstoß geben und damit eine kritische Selbstüberprüfung der eigenen Arbeit anregen.

Funding Open Access funding enabled and organized by Projekt DEAL.

Open Access Dieser Artikel wird unter der Creative Commons Namensnennung 4.0 International Lizenz veröffentlicht, welche die Nutzung, Vervielfältigung, Bearbeitung, Verbreitung und Wiedergabe in jeglichem Medium und Format erlaubt, sofern Sie den/die ursprünglichen Autor(en) und die Quelle ordnungsgemäß nennen, einen Link zur Creative Commons Lizenz beifügen und angeben, ob Änderungen vorgenommen wurden.

Die in diesem Artikel enthaltenen Bilder und sonstiges Drittmaterial unterliegen ebenfalls der genannten Creative Commons Lizenz, sofern sich aus der Abbildungslegende nichts anderes ergibt. Sofern das betreffende Material nicht unter der genannten Creative Commons Lizenz steht und die betreffende Handlung nicht nach gesetzlichen Vorschriften erlaubt ist, ist für die oben aufgeführten Weiterverwendungen des Materials die Einwilligung des jeweiligen Rechteinhabers einzuholen.

Weitere Details zur Lizenz entnehmen Sie bitte der Lizenzinformation auf http://creativecommons.org/ licenses/by/4.0/deed.de. 